\title{
EDITORIAL
}

nature

cell biology

\section{Does size matter?}

\section{The format of papers in this journal is similar to comparable journals.}

When it comes to text, Nature has always quite consciously followed a tight publication format. It may surprise some that the reason for this is not to save on paper (or trees), but instead to make papers more accessible to a broad readership. To some, more text implies more accessibility. We beg to differ: well-crafted, accurate and concise text is the most efficient vehicle to communicate complex scientific information. Rambling repetitive text, which gets stuck in unnecessary detail or history, serves only to confuse. It also puts off the general reader, who has limited time to read beyond the literature immediately relevant to their research. Of course, it is necessary to achieve the right balance so that all essential information can be provided and understood by as wide a readership as possible.

Catering for a slightly more specialist audience, the Nature Research Journals have a more relaxed set of format requirements that, in our opinion, give adequate space for a detailed contextualization and discussion of the data. Nevertheless, format is one of the most frequent reasons given for shying away from submission — or indeed to explain a lack of experimental data. These authors fail to appreciate that Nature Cell Biology is actually comparable to alternative venues for publication when data and text presented in supplementary information is included (as it should be, given that supplementary information is a fully peer reviewed integral part of Nature Cell Biology papers and is therefore included in the main PDF file): Nature Cell Biology articles contain an average 9,006 words (7,903 in the main paper), with 11.5 figures (6.4 in the main paper) composed of 46.9 individual panels (26.8 in main paper). Compare this to The Journal of Cell Biology - 9,472 words average text length $(9,248$ in main paper), 10.1 figures (8.4 in the paper) with 31 panels (24.9 in the paper); or Cell - 11,042 words (10,188 in paper), 11.7 figures (6.7 in paper) with 48.3 panels (33.5 in paper). Importantly, Cell papers contain approximately the same amount of data as Nature Cell Biology articles, and The Journal of Cell Biology papers contain somewhat less. Cell papers contain 229 words per display panel, whereas Nature Cell Biology articles contain 192 and The Journal of Cell Biology papers are 5\% longer than Nature Cell Biology articles. Of course, Nature Cell Biology letters are shorter (5,961 words and 8.0 figures, of which 4.7 are in the main paper, and 33.5 panels with 25 in the main paper), but articles represent approximately $30 \%$ of our papers, and we will not cut down a paper to letter format if this damages the contents ${ }^{\mathrm{a}}$.

A valid criticism of the terse format of Nature has been the occasional difficulty in reproducing data based on the methods provided. It is certainly true that dependence on the citation of previous studies using similar methods can lead to inaccuracies. At the Nature research titles, we do not enforce a strict limitation of the methods section for this reason. Notably, Nature has also addressed this issue by publishing bipartite methods henceforth: a short summary in the main paper and an online document with extended methods in the supplementary information.

We do hope this addresses any rumblings about excessive format restrictions.

\section{Nature China}

\section{Nature Publishing Group is launching a web portal to keep abreast of the best research emerging from China.}

Cell biology research in China is a relatively young plant that has been growing apace with the country's rapid global development. Indeed, the plant is also increasingly bearing fruit - not just in the shape of new and well-equipped research facilities such as the SIBS's Institute of Biochemistry and Cell Biology, which would not be out of place in Shanghai's famous architectural show room along the Huangpu River, but also in terms of high profile papers. China's publication rate in peer reviewed journals has risen eightfold in the past decade to rival that of the UK and Japan. Importantly, the number of papers with in excess of 20 citations has also risen tenfold, although in terms of absolute levels they remain in the hundreds, and biomedical science represent less than $20 \%$ of mainland China's high-impact papers. We have also noticed a similar tenfold rise in submissions from China to this journal over the past seven years, which we hope will be reflected in a proportional representation in print. However, to put things into perspective, current submissions from China amount to $2 \%$ of total submissions to Nature Cell Biology.

For over ten years Nature Publishing Group has maintained the Nature Japan Gateway, which provides Japanese language access to content across the Nature titles, as well as jobs advertisements to over 120,000 registrants who can chose from fifteen customized email alerts. More recently, similar gateways for Korea and China were launched, and despite the considerably lower number of registrants, these services already receive high web-traffic.

The new Nature China portal has a dedicated Hong Kong-based editor, who will provide accessible short summaries in English and Chinese to a weekly updated selection of top research papers across all fields and across the literature from mainland China and Hong Kong. The site also allows users to recommend papers, and the community is encouraged to comment and vote on these papers as part of a global virtual journal club. In addition, there will be an open-access archive linking to papers from China published across the Nature journals in the last five years. The site has a local mirror, which will provide Chinese readers with efficient access to the articles featured.

So whether you are a researcher in China or simply interested in Chinese science, do sign up to the weekly e-mail alert and contribute your selection of the best research China has to offer when the full site launches in late April.

${ }^{a}$ The data is based on ten randomly sampled recent papers from each journal. Text counts exclude figure legends. 